PHYSICAL REVIEW B, VOLUME 64, 179902(E)

\title{
Erratum: Resonant-cavity-induced phase locking and voltage steps in a Josephson array [Phys. Rev. B 63, 144522 (2001)]
}

\author{
E. Almaas and D. Stroud \\ (Published 4 October 2001)
}

DOI: 10.1103/PhysRevB.64.179902

PACS number(s): 05.45.Xt, 05.45.- a, 74.40.+k, 99.10.+g

In the last full paragraph in the left-hand column of p. 144522-2, in the sentence beginning "Specifically, we may add a term to $H \ldots$, " the symbol $\phi_{j}$ should be replaced by $\gamma_{j}$. Similarly, in the last sentence of that paragraph, $\phi_{j}$ should be replaced by $\gamma_{j}$. When the equations of motion resulting from these changes are solved numerically, the results are substantially improved. The evidence for the conclusions of the paper is thus significantly strengthened. Complete numerical results will be presented elsewhere. 